\title{
Magnetic Properties of bcc Iron Surfaces and the Influence of the Chemical Environment: Electronic Structure Calculations
}

\author{
Guntram Fischer ${ }^{1}$, Iann C. Gerber ${ }^{1}$ \\ 1 Université de Toulouse; INSA, UPS, CNRS; LPCNO, 135 Avenue de Rangueil, \\ 31077 Toulouse, France \\ E-mail: igerber@insa-toulouse.fr
}

\begin{abstract}
We have investigated the magnetic properties of low-indexed iron surfaces and the influence of the chemical environment on these properties. We considered the (100), (110), (111), (211), and (310) surfaces, both, bare and with ligands adsorbed. The ligands were chosen according to possible chemical environments, being $\mathrm{H}, \mathrm{Cl}, \mathrm{HCl}$, $\mathrm{NH}_{3}, \mathrm{NH}_{4} \mathrm{Cl}$, or $\mathrm{CH}_{3} \mathrm{COOH}$. We find an increased magnetization at all bare surfaces. Upon $\mathrm{H}$ adsorption the magnetization is generally reduced, but still above the bulk value. All other ligands and dissociated parts of them alter the magnetic properties of the surfaces only weakly. Our calculations do not indicate that ligands are responsible for experimental observations of Fe nanoparticles with average magnetizations below the bulk value.
\end{abstract}

PACS numbers: $75.50 . \mathrm{Bb}, 75.70 .-\mathrm{i}, 75.75 .-\mathrm{c}$

Keywords: Surface, Magnetism, Iron, Ligands, Adsorption, Density Functional Theory

Submitted to: J. Phys.: Condens. Matter 


\section{Introduction}

Magnetic nanoparticles (NP) belong to the group of nanostructured materials which offer a wide range of possible applications in different fields, such as spintronics, catalysis, or medicine. [1-5] They are of special interest in the medical treatment of cancer in so-called hyperthermiae. [6,7] In this context a high magnetic moment $(m)$ per NP and, therefore, a high $m$ per atom are desirable.

Iron is of special interest as a material for NP due to its relatively moderate cost and bio-degradability. More importantly, it offers favorable magnetic properties such as a large atomic magnetic moment and strong magnetic interaction, which enable stable magnetic nanosystems and their accessibility and efficient manipulation even at very small scales. The magnetic properties of iron NP have, therefore, been studied intensively throughout the last years. [8] In particular, it has been verified for pure Fe clusters consisting of only few up to several hundreds of atoms experimentally $[9,10]$ as well as theoretically [11-14] that pure iron NP have, in average, an increased atomic $m$ which converges to the bulk value for increasing cluster sizes. The high $m$ can be explained by the reduced coordination number $\left(N_{C}\right)$ of the atoms at the surface. [15] Furthermore, the magnetic coupling between the atoms is largely ferromagnetic. $[10,14]$ However, since pure iron is very reactive, the surface of a pure NP will, depending on its surroundings, be quickly polluted by atoms or molecules. Therefore, it is important to investigate the influence of the chemical environment on the magnetism.

One possibility to prevent unwanted adsorption is coating with noble metals, oxides, or other surfactants. [5] Such treatment, however, alters the properties of the NP, and experiments show that the average magnetization is lowered below the bulk value of $2.21 \mu_{B}$. $[16,17]$ Aside from that, depending on the specific method, the NP may already get into contact with other materials during the preparation, which is the case for the synthesis of iron NP involving the decomposition of organometallic compounds. [7,18-21] It has indeed been observed that chemically synthesized Fe NP have magnetizations slightly below the bulk value, $[7,18,19]$ although, as stated above, it would be expected that the contrary is the case, i.e. a NP magnetization higher than in bulk Fe. Such chemical synthesis reactions are likely to involve the presence of nitrogen, chlorine, and hydrogen and their compounds.

In order to obtain results that are applicable to a large variety of shapes and sizes of NP it is reasonable to not study adsorption at the NP directly, but to rather investigate the effect of ligands on infinitely extended Fe surfaces. This holds especially for NP of dimensions of several $\mathrm{nm}$, i.e. such showing facets with large surface areas. This is the case for a large part of different synthesis processes $[5,7,16,22]$ and applications $[5,17]$ of NP, where the chemical environment and ligand effects may play an important role, and it outweighs the drawbacks of neglecting the effects of edges and corners.

Here we present first principles electronic structure calculations on the five lowindex Fe surfaces that have the lowest surface energies according to previous theoretical studies. [15] These surfaces are the (100), (110), (111), (211), and (310). As ligands 
we chose $\mathrm{H}, \mathrm{Cl}, \mathrm{HCl}, \mathrm{NH}_{3}, \mathrm{NH}_{4} \mathrm{Cl}$, and $\mathrm{CH}_{3} \mathrm{COOH}$, since these species are likely to be present during the synthesis of NPs in mild conditions. The respective adsorption geometries are illustrated in the supplemental information. A profound discussion on the adsorption behaviour of these compounds on the different facets, considering thermodynamic and vibrational effects, is given elsewhere. [23] In the present work we have studied the magnetic moment distribution of the five mentioned, bare surfaces and have investigated the influence of the chemical environment on the respective surface magnetization.

The paper is structured as follows. In Section 2 we give details on the parameters and the setup used for the electronic structure calculations as well as information on the theoretical concepts used to analyze the results. All results are then presented and discussed in Section 3. The paper closes with a summary.

\section{Theoretical Details}

\subsection{Electronic Structure Calculations}

We performed density functional theory (DFT) [24,25] calculations in the generalized gradient approximation (GGA) in the Perdew-Burke-Ernzerhof (PBE) parametrisation [26]. This was done within the Vienna ab initio simulation package (VASP) $[27,28]$ using the projector augmented wave method. [29] The energy cut-off was chosen to be $400 \mathrm{eV}$.

For the determination of the minimum energy Fe bulk lattice constant $a_{\min }$ we used a bcc Fe unit cell and a $k$-mesh of $20 \times 20 \times 20$ points in the full Brillouin zone. Our value of $2.84 \AA$ for $a_{\text {min }}$ agrees well with the experimental [30] value of $2.87 \AA$ and also with those from previous theoretical investigations. [15,31] Furthermore, the agreement between the calculated bulk magnetic moments per Fe atom is very good as well, being $m_{\text {bulk }}=2.21 \mu_{B}$ in our work and, for example, $2.20 \mu_{B}$ in Reference [15].

The surfaces were modelled using $2 \times 2$ surface unit cells with a slab thickness of 9 monolayers (ML), resulting in 36 Fe atoms per cell; for the (310) surface $11 \mathrm{ML}$ were used resulting in $44 \mathrm{Fe}$ atoms. The cell sizes in $z$-direction have been carefully checked to avoid spurious interaction between the periodic slab images. This resulted in a vacuum region between the slabs larger than $16 \AA$ for all five surfaces. We used a $k$-mesh of $6 \times 6 \times 1$ points for structural relaxation and of $9 \times 9 \times 1$ points for the precise calculation of the total energy of the relaxed structures, the magnetic properties, and the atomic Bader charges. [32,33] The expansion of the plane waves was cut off at $400 \mathrm{eV}$. For the calculation of the isolated ligands a cubic cell of dimensions $20 \times 20 \times 20 \AA^{3}$ and a $2 \times 2 \times 2 k$ mesh were used.

The central three (five in case of (310)) ML were kept fixed at the minimum energy lattice $a_{\text {min }}$ constant. The geometric relaxation of the three outmost MLs of the surface cells was done applying the residual minimization method, [34] where structural convergence was assumed to be achieved when the forces acting on all atoms were less 
than $0.02 \mathrm{eVA}^{-1}$.

For all investigated ligands we tested several high symmetry positions on each surface as adsorption sites. They are illustrated in Figure 1. In addition to the position,

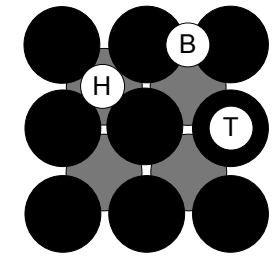

(a) (100)

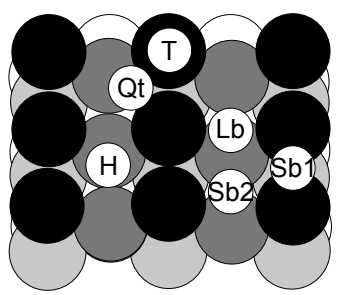

(d) (211)

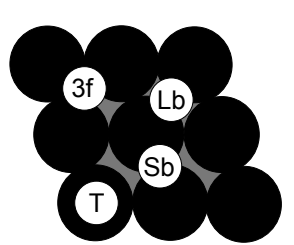

(b) (110)

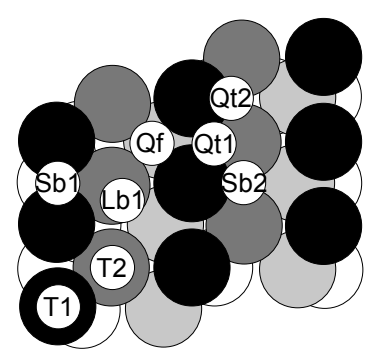

(e) (310)

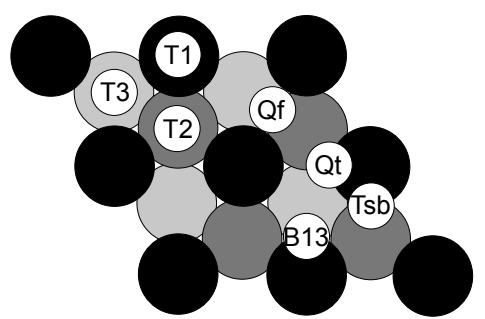

(c) (111)

Figure 1. Top views of the five investigated $2 \times 2$ surface cells. The Fe atoms in the first (black), second (dark grey), third (light grey), and deeper (white) MLs are shown with the possible high symmetry adsorption sites of each surface (small white). These are: 1(a) B - bridge, H - hollow, T - top; 1(b) 3f - (quasi) 3-fold hollow, Lb - long bridge, Sb - short bridge, T - Top; 1(c) B13 - bridge between Fe in 1st and 3rd layer, Qf - quasi four-fold, Qt - quasi three-fold, T1 (T2, T3) - on top of Fe in the 1st (2nd, 3rd) ML, Tsb - top-shallow bridge; 1(d) H - hollow, Lb - long bridge (between 1st ML Fe atoms), Qt - quasi 3 fold hollw, Sb - short bridge, Sb2 - short bridge between 2nd ML, T - top; 1(e) Lb1 - long bridge between 1st ML Fe, Qf - quasi four-fold, Qt1 (Qt2) - quasi three-fold with two Fe atoms of 1st (2nd) ML, Sb1 (Sb2) - short bridge between 1st (2nd) ML Fe, T1 (T2) - top of 1st (2nd) ML Fe.

the number $n$ of ligand atoms or molecules per $2 \times 2$ surface cell was used as a parameter as well. Eventually, for each surface and ligand, the specific $n=n_{0}$ and the adsorption sites minimizing the surface energy of the specific system were further evaluated with respect to their magnetic properties. Note that different numbers of adsorbed ligands on the surfaces result in different concentration values since the surface area of each surface cell differs from the other, as indicated in Figure 1. The values are listed in Table 1.

\subsection{Surface Energies}

In order to discuss the magnetic properties of any system it is necessary to study the configurations that are physically meaningful. In the present case this means that the investigated Fe surfaces, bare or decorated, must be stable by thermodynamical means. The criterion for the stability of each surface is the surface energy $\gamma_{h k l}$, where $h k l$ 
denotes the respective Miller index. For a bare surface it is necessary to calculate the total energy $E_{h k l}^{N}$ of the Fe slab consisting of $N$ Fe atoms, and the total energy of one Fe atom in the bulk phase, $E_{\text {bulk }}$. The $\gamma_{h k l}^{0}$, where the upper index 0 refers to the bare surface, are then obtained via

$$
\gamma_{h k l}^{0}=\frac{1}{2 A_{h k l}}\left(E_{h k l}^{N}-N E_{\mathrm{bulk}}\right),
$$

where $A_{h k l}$ is the surface area and the factor $1 / 2$ corresponds to the fact that the slab has two surfaces. Note that this definition is formally valid only at zero Kelvin. However, since the Fe atoms remain in the condensed phase throughout the investigated process, it can be assumed that effects resulting from finite temperatures cancel out and, thus, that Equation (1) can be extended to finite temperatures. [35]

For a surface $h k l$ dressed by $n$ ligands $L$ the surface energy is defined as

$$
\gamma_{h k l}=\gamma_{h k l}^{0}+\frac{\Delta_{a} G_{h k l, n L}(T, p)}{2 A_{h k l}} .
$$

Here, $\Delta_{a} G_{h k l, n L}(T, p)$ is the Gibbs free energy of adsorption of $n$ ligands $L$ on the surface $(h k l)$, i.e. of the reaction $(h k l)+n L \rightarrow n L *(h k l)$. It contains the electronic, thermodynamic and vibrational contributions to the energy. [23] In the case of zero Kelvin and no pressure, the latter two equal zero and only the electronic contributions are considered, resulting in

$$
\Delta_{a} G_{h k l, n L}(T, p)=E_{\mathrm{ads}}=E_{n L *(h k l)}-E_{(h k l)}-n E_{L}^{\mathrm{el}} .
$$

$E_{\text {ads }}$ is called adsorption energy and $E_{L}^{\text {el }}$ represents the chemical potential of the isolated ligands L, obtained as described in Section 2.1. Note that for the calculation of the contribution of one $\mathrm{Cl}$ atom, $E_{\mathrm{Cl}}^{\mathrm{el}}(0,0)$, we evaluated the difference $E_{\mathrm{HCl}}^{\mathrm{el}}(0,0)-$ $1 / 2 E_{\mathrm{H}_{2}}^{\text {el }}(0,0)$. We assume that $\mathrm{HCl}$ is the only source of $\mathrm{Cl}$ atoms.

Neglecting temperature and vibrational effects leads to rather rough estimates for the surface energies and may result in too low minimum energy coverage values. However, with the purpose of the present paper being the study of magnetic properties, the $\gamma_{h k l}$ are, firstly, used to indicate possible energetical preferrences of certain facets and, secondly, to mark maximum coverage values for the ligands. The first can be expected not to be considerably affected by thermodynamic and vibrational contributions. Regarding the second issue, since ignoring of the thermodynamic and vibrational effects lowers surface energies, [23] one will in the worst case consider configurations with too many adsorbed ligands. This, however, will not affect observed trends and results for the thermodynamically meaningful values. For a comprehensive discussion of the adsorption behaviour of the investigated ligands at finite temperatures we refer the reader to our previous paper. [23]

\subsection{Magnetic Properties}

The most intuitive quantity describing the magnetic properties is the atomic moment $m$ located at each atom. In order to estimate the increase of $m$ for each surface, we have 
calculated the total additional moment induced at the surface, with respect to the bulk value, by the three topmost layers. The choice of the three top layers is reasonable as it is those that were allowed to relax and as they contain the major contributions to the surface magnetization. The increase is defined via

$$
\Delta m_{13}=\sum_{i=1}^{n_{\mathrm{Fe}}}\left(m_{1}^{i}+m_{2}^{i}+m_{3}^{i}\right)-3 n_{\mathrm{Fe}} m_{\mathrm{bulk}},
$$

where $n_{\mathrm{Fe}}$ is the number of Fe atoms per layer in the slab $\left(n_{\mathrm{Fe}}=1\right.$ (4) in case of the $1 \times 1(2 \times 2)$ surface cell $)$ and $m_{1,2,3}^{i}$ is the magnetic moment of the $i$-th Fe atom in the first, second, or third layer, respectively.

Only for comparison we have as well estimated the total increase of $m$ by calculating the additional moment induced at the whole surface,

$$
\Delta m_{\mathrm{surf}}=m_{\mathrm{surf}}^{\mathrm{tot}}-n_{\mathrm{Fe}} m_{\mathrm{bulk}} .
$$

In Equation (5) $m_{\text {surf }}^{\text {tot }}$ is the total moment in the considered surface cell and $n_{\mathrm{Fe}}$ the number of respective Fe atoms in that cell. Note that Equation (5) implicitely includes the magnetic moment of the ligands, which, however, is smaller than $0.15 \mu_{B}$ in all adsorbed configurations and, furthermore, identical zero in all cases of isolated molecules. Note as well, that compared to $\Delta m_{13}$, the convergence of $\Delta m_{\text {surf }}$ with respect to the number of layers is slow, in particular for the more open (111) and (310) surfaces. [15] We have, therefore, used $\Delta m_{\text {surf }}$ of Equation (5) only by means of verifying the results of $\Delta m_{13}$ of Equation (4). By doing so, all trends presented in the following sections are confirmed.

\section{Results and Discussion}

\subsection{Bare Surfaces}

The calculation of the surface energies of the bare surfaces $\gamma_{h k l}^{0}$, Equation (1), yields results that are in very good agreement with previous theoretical works. $[15,36,37]$ As expected [15] the magnetic moment of the Fe atoms near the surface is enhanced, compared to the bulk value of $m_{\text {bulk }}=2.21 \mu_{B}$ per atom. This holds for all surfaces investigated, as shown in Table 1 . The difference of the surface atomic $m$ in dependence on the depth of the respective atom is shown in Figure 2. A strong increase of $m_{1}$, the moment at the topmost layer atom, can be observed for all surfaces, which is in agreement with previous results. [15] Furthermore, the numerical values for $m_{1}$, given in Table 1, are within around $1 \%$ identical to those of Reference [15]. Table 1 shows as well, that the increase of $m_{1}$ is indirectly related to $N_{C}$ of the topmost Fe atom, a fact that has as well already been observed. [11,15] Although the differences between the $m_{1}$ in the (100), (111), and (310) case are only small, it is interesting to note that there is a relation between $m_{1}$ and the distance of the topmost Fe and its nearest neighbours (NN) after relaxation, namely that $m_{1}$ increases with distance: $2.84 \mu_{B}<2.88 \mu_{B}<2.94 \mu_{B}$ with $2.35 \AA<2.38 \AA<2.45 \AA$ for the (111), (310), and (100) cases, respectively. 


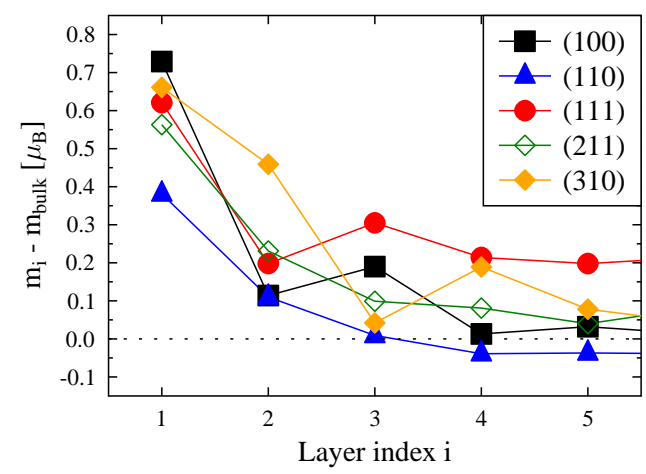

Figure 2. Difference between the atomic magnetic moment of the 5 topmost layers of each investigated bare surface and the bulk value of iron, indicated by the dotted line.

Table 1. Surface energies $\gamma_{h k l}^{0}$, corresponding surface areas $A$, and magnetic moment $m_{1}$ of the topmost Fe atom. The coordination number $N_{C}$, i.e. number of nearest neighbors, explains well the different quantities of $m_{1}$. Shown as well are the enhancement $\Delta m_{13}$ of the magnetic moment at the surface per Fe atom, with respect to the bulk value, and the resulting magnetization density increase $\Delta m_{13} / A$.

\begin{tabular}{cccccc}
\hline$h k l$ & $(100)$ & $(110)$ & $(111)$ & $(211)$ & $(310)$ \\
\hline$\gamma_{h k l}^{0}\left[\mathrm{~J} / \mathrm{m}^{2}\right]$ & 2.47 & 2.41 & 2.65 & 2.56 & 2.50 \\
$A_{h k l}\left[\AA^{2}\right]$ & 32.29 & 22.83 & 55.92 & 39.54 & 51.05 \\
$m_{1}\left[\mu_{B}\right]$ & 2.94 & 2.60 & 2.84 & 2.78 & 2.88 \\
$N_{C}$ & 4 & 6 & 4 & 5 & 4 \\
$\Delta m_{13}\left[\mu_{B}\right]$ & 0.85 & 0.38 & 0.76 & 0.79 & 1.12 \\
$\Delta m_{13} / A\left[\mu_{B} / \AA^{2}\right]$ & 0.105 & 0.086 & 0.054 & 0.080 & 0.088 \\
\hline
\end{tabular}

Concerning $N_{C}$ a similar trend seems to hold for the second Fe layer. As can be seen in Figure 2, the magnetic moment is lowest for the (100) and (110) surfaces having a $N_{C}$ of 8 . In the case of the (111) and (211), $N_{C}$ is 7 . The Fe atom in the second layer at the (310) surface has the lowest coordination, $N_{C}=6$, and as well the highest $m$.

The results for $\Delta m_{13}$ are also given in Table 1 . The increase with respect to the bulk case is most pronounced for the (310) surface. For the (111) surface, there is a considerable moment at the 3rd layer iron atom, being $0.30 \mu_{B}$, see as well Figure 2 . The reason for this is the oscillating magnetization density, which is a metallic property, in combination with the tight stacking of the atoms perpendicular to the surface. The distance between the first and third layer is only $1.43 \AA$. One can see from Figure 2 that considering as well the moments of the 3rd layer Fe atoms generally leads to an increase of all surface magnetizations with the exception of the (110) case.

While this increase per atom is largest for the (310) surface, the division of $\Delta m_{13}$ by the surface area $A_{h k l}$ shows that the magnetization density will be largest at the (100) facet due to its relatively small size. Thus, we find that the increase in magnetic moments is strongest at the (100) surface. This suggests in return that naked Fe nanocubes 
Table 2. Coverage values $n_{0}$ (in atoms per $2 \times 2$ surface cell) resulting in the minimum surface energies $\gamma_{h k l}$ upon $\mathrm{H}$ adsorption, the adsorption energy $E_{\text {ads }}$ for one $\mathrm{H}$, the respective adsorption sites, see Figure 1, and the distance $d$ between the hydrides and the nearest Fe neighbour. The sites hold for $n=1$ as well as for $n_{0}$, the values for $d$ are for the case of $n=n_{0}$ and differ from those for $n=1$ by maximally $0.05 \AA$ due to different magnitudes of relaxation for different $n$.

\begin{tabular}{cccccc}
\hline$h k l$ & $(100)$ & $(110)$ & $(111)$ & $(211)$ & $(310)$ \\
\hline$n_{0}$ & 4 & 4 & 8 & 4 & 4 \\
$\gamma_{h k l}^{n_{0}}\left[\mathrm{~J} / \mathrm{m}^{2}\right]$ & 1.66 & 0.59 & 1.54 & 1.67 & 1.69 \\
$E_{\text {ads }}^{n=1} / \mathrm{eV}$ & -0.40 & -0.70 & -0.57 & -0.55 & -0.65 \\
Site & $\mathrm{H}$ & $3 \mathrm{f}$ & $\mathrm{Tsb}(\mathrm{Qf})$ & $\mathrm{Qt}$ & $\mathrm{Qt} 1$ \\
$d[\AA]$ & 1.74 & 1.73 & $1.63(1.66)$ & 1.77 & 1.78 \\
\hline
\end{tabular}

presenting (100) facets, are of favorable shape with a large yield of magnetic moment. This is because, of all possible Wulff constructions with surface area $A_{h k l}$ and volume $V_{h k l}$, they represent the one with the highest $A_{h k l} / V_{h k l}$ ratio.

\subsection{H Adsorption}

Table 2 summarizes the adsorption energetics and thermodynamic equilibrium configurations. One can identify a strong preference for the (110) facet. More details are given in Ref. [23]. Along with the $\mathrm{H}$ adsorption goes a charge transfer away from the surface Fe atoms of a magnitude between 0.29 and $0.40 e$ per ligand atom, independent on the surface and number of adsorbed species. The transfer is more pronounced for low coverage values and large surface unit cells, as the interaction between the ligands is small in this case and, thus, the interaction between $\mathrm{H}$ and Fe stronger. Generally, this charge transfer means that all $\mathrm{H}$ adsorbed at the surfaces is negatively charged.

The effect of an increasing $H$ coverage value on the magnetization up to the $n_{0}$ listed in Table 2 is summarised in Figure 3. It shows a monotonous decrease with growing

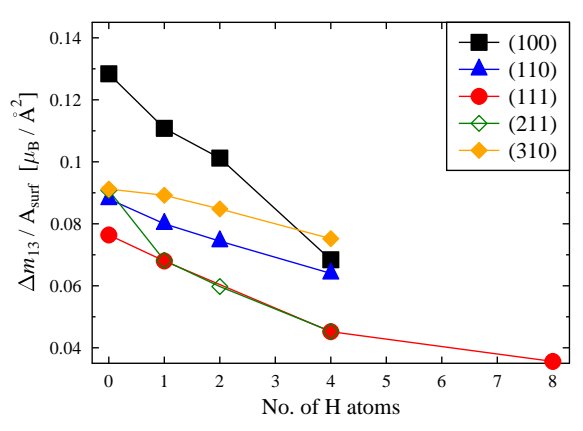

Figure 3. The change of $m$ of the three topmost Fe atoms upon $\mathrm{H}$ adsorption up to the coverage values given in Table 2 .

ligand number. The local density of states (LDOS) of one of the topmost Fe atoms 
in case of the bare and the fully $\mathrm{H}$ covered (100) surface are compared in Figure 4. The presence of $\mathrm{H}$ shifts the states at the Fermi level $E_{F}$ in the minority spin channel

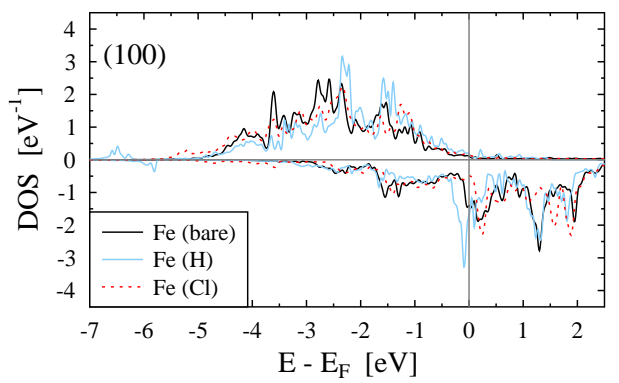

Figure 4. The LDOS of the topmost Fe layer per Fe atom for the bare, $\mathrm{H}-$, and $\mathrm{Cl}$ covered (100) surface. Majority spin states are in the upper panel, minority states in the lower one. The adsorption configurations of the minimum surface energy have been chosen.

below $E_{F}$. This hints to a strong interaction between the $\mathrm{H} s$ and the Fe $d$ electrons, as confirmed by Figure 5 showing that the hybridization between the Fe $d$ states around $6 \mathrm{eV}$ below $E_{F}$ and the $\mathrm{H}$ electrons is large, as both peaks have similar magnitudes. As a consequence the unoccupied states in the minority spin channel of the bare surface are pushed below $E_{F}$ and become partially occupied, which is shown in Figure 4.

At first sight, the decrease of $m$ at the surface seems to be in contradiction to observations of $\mathrm{H}$ adsorption on thin films [38,39] and iron clusters, [40] where an increase of the magnetization was found. This discrepancy, however, can be explained. Firstly, our slab approach, with a large number of MLs, would not be appropriate to describe the experimental samples of both very thin films (2 ML in Reference [39]) and nano clusters. [40] Secondly, the films were deposited on glass [38] or copper. [39] Charge transfer can occur especially between the copper and the iron and may, thus, strongly influence the magnetic properties of the Fe film. Third, the structure of the films was polymorph [38] or fcc. [39] Our disagreement with a bond order - rigid band model, which was used to explained the above experimental observations, is most likely due to the assumption of a bulk-like magnetic structure for clusters and surfaces. [41] As found and demonstrated in Figure 2, this is not valid.

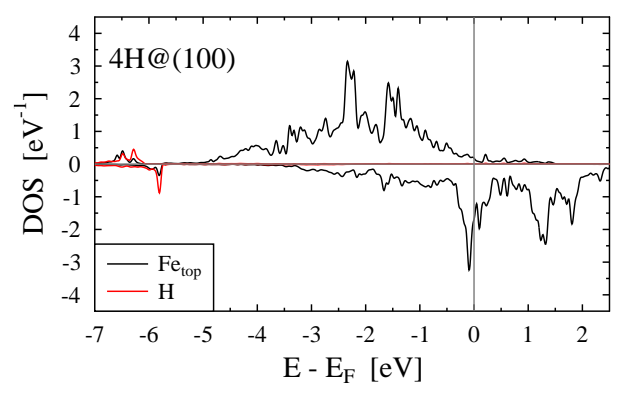

Figure 5. The DOS per atom at a H ligand and at a topmost surface Fe atom. 
Table 3. The respective $\mathrm{Cl}$ coverage values $n_{0}$ (in atoms per $2 \times 2$ surface cell) leading to the lowest surface energy values, the respective surface energies $\gamma_{h k l}$, the adsorption energy $E_{\text {ads }}$ for one adsorbed $\mathrm{Cl}$, and the adsorption sites of one $\mathrm{Cl}$ atom (and, if being different, of the respective minimum configurations).

\begin{tabular}{cccccc}
\hline$h k l$ & $(100)$ & $(110)$ & $(111)$ & $(211)$ & $(310)$ \\
\hline$n_{0}$ & 2 & 2 & 4 & 2 & 4 \\
$\gamma_{h k l}^{n_{0}}\left[\mathrm{~J} / \mathrm{m}^{2}\right]$ & 0.98 & 0.92 & 0.84 & 1.19 & 0.78 \\
$E_{\text {ads }}^{n=1} / \mathrm{eV}$ & -1.46 & -1.67 & -1.62 & -1.69 & -1.74 \\
Site & $\mathrm{H}(\mathrm{B})$ & $\mathrm{Lb}$ & $\mathrm{T} 2$ & $\mathrm{Sb}$ & $\mathrm{Qt1}$ \\
\hline
\end{tabular}

The linear dependence of the magnetization on the number of ligand hydrides, see Figure 3, suggests that the ligands themselves do not interact significantly with each other. For the (111) surface, the reduced slope at more than 4 ligands is caused by, on one side, the high concentration of $\mathrm{H}$ leading to electronic interaction between them and, thus, to a weakening of the coupling to the surface. On the other side, the fact that the four $\mathrm{H}$ atoms at the Qf site are closer to the Fe surface and, in fact, to the Fe atoms in the second ML than to those in the first ML, [23] plays a role. The electronic interaction of these $\mathrm{H}$ with the second $\mathrm{ML}$ will be stronger than that with the first ML. Since this interaction weakens $m, \Delta m_{13}$ will be smaller for the $\mathrm{H}$ closer to the less magnetic second ML of Fe.

The lowering effect of $\mathrm{H}$ ligands on the surface magnetization can, however, not fully explain the experimental observations mentioned above, $[7,19]$ because the surface magnetization at each surface still exceeds the bulk value, as shown in Figure 3. Since it was shown that diffusion of $\mathrm{H}$ atoms from the surface into the bulk is likely to occur at certain surfaces [31] we have as well tested configurations containing additional $\mathrm{H}$ atoms. Indeed we find that $1 \mathrm{H}$ atom at the tetragonal subsurface site below the (100) surface [31] decreases the magnetization more strongly than a $\mathrm{H}$ ligand at the surface. For the ratio $\Delta m_{13} / A$ we obtain $0.102 \mu_{B} / \AA^{2}$, which is around $10 \%$ lower than the value of $0.111 \mu_{B} / \AA^{2}$ for $1 \mathrm{H}$ ligand, see Figure 3 . Adding further subsurface $\mathrm{H}$ atoms, however, does not lead to a reduction of $\Delta m_{13}$ to negative values. For example, assuming 4 ligands $\mathrm{H}$ and 4 subsurface $\mathrm{H}$, results in $0.072 \mu_{B} / \AA^{2}$ for $\Delta m_{13} / A$, which is of the same order as the value for 4 ligands, namely $0.068 \mu_{B} / \AA^{2}$, representing the minimum surface energy. Note that the configuration with 4 ligands and 4 subsurface $\mathrm{H}$ atoms is thermodynamically not stable.

\subsection{Cl Adsorption}

Table 3 shows that chlorine atoms are likely to adsorb on iron as the listed $\gamma_{h k l}$ are significantly lower than those of the bare surfaces. The respective adsorption sites at each surface are, together with the respective $n_{0}$, listed underneath the $\gamma_{h k l}$.

The magnetic effects of the chlorine adsorption, up to the minimum surface energy coverage as listed in Table 3, are summarised in Figure 6. It shows that $\mathrm{Cl}$ adsorption 


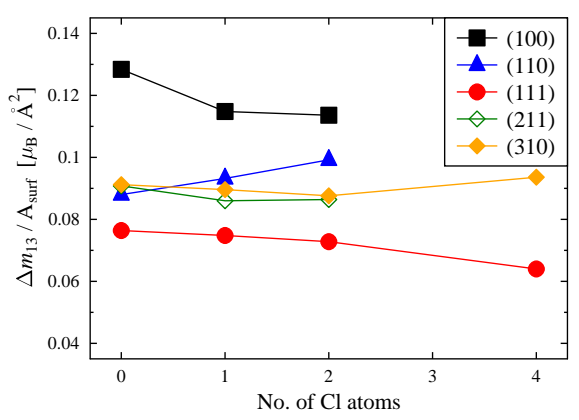

Figure 6. Magnetic moment of the three top Fe atoms, with respect to the bulk value, upon $\mathrm{Cl}$ adsorption up to the coverage values given in Table 3 .

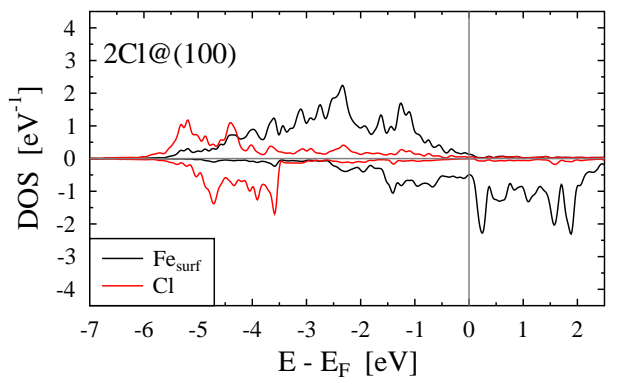

Figure 7. The LDOS of $\mathrm{Cl}$ and the topmost Fe atom of the (100) surface with two $\mathrm{Cl}$ atoms adsorbed.

has only a very weak effect on the surface magnetism. This is surprising since there is again a charge transfer away from the surface to the $\mathrm{Cl}$ ligands, as the negative charge per $\mathrm{Cl}$ ligand is between $0.50 e$ and $0.63 e$ for all surfaces. The minimum value holds for the case of 2 ligands at the (110) surface, the maximum for $n=1$ in the (100) case. The ionic character is generally more pronounced for low coverage values and large surface unit cells, as the interaction between the ligands is small in that case. Due to the larger charge transfer one might expect an even stronger reduction of $m$ compared to the case of Hydrogen adsorption.

The relative independence of the magnetization on the number of ligands can be explained by looking at the characteristics of the LDOS of the topmost Fe layer in the case of $2 \mathrm{Cl}$ atoms adsorbed on the (100) surface in Figures 4 and 7 . The discussed characteristics are also valid for the other surfaces. Obviously, hybridization between the $\mathrm{Cl}$ and the top Fe states occurs, to small extends, only in the majority channel, as can be seen in Figure 7. This is most likely caused by the large distance between the $\mathrm{Fe}$ and $\mathrm{Cl}$ atoms of more than $2.3 \AA$ at all surfaces and, thus, being at least more than $0.5 \AA$ greater than that between the Fe and $\mathrm{H}$ atoms. The hybridization causes the majority states to be slightly pushed up in energy. Together with the withdrawal of electrons from the Fe towards the $\mathrm{Cl}$, this lets both minority as well as majority states become unoccupied, see Figure 4, resulting in the weak change of magnetization. 


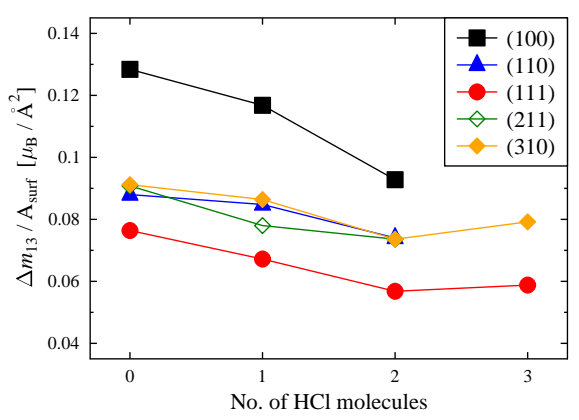

Figure 8. Magnetic moment of the three top $\mathrm{Fe}$ atoms upon $\mathrm{HCl}$ adsorption, with respect to the bulk value, up to the coverage values given in Table 4.

\section{4. $\mathrm{HCl}$ Adsorption}

Table 4 summarizes the calculations on the minimum energy configuration for all five surfaces. It shows that the respective minimum surface energies $\gamma_{h k l}^{n_{0}}$ are well below

Table 4. $\mathrm{HCl}$ minimum surface energy coverage values $n_{0}$ (in molecules per $2 \times 2$ surface cell), the respective minimum surface energy $\gamma_{h k l}^{n_{0}}$, the adsorption energy of one dissociated $\mathrm{HCl}$, and the respective adsorption sites in the form $X-Y$ with $X(Y)$ being the site of $\mathrm{H}(\mathrm{Cl})$.

\begin{tabular}{cccccc}
\hline$h k l$ & $(100)$ & $(110)$ & $(111)$ & $(211)$ & $(310)$ \\
\hline$n_{0}$ & 2 & 2 & 3 & 2 & 3 \\
$\gamma_{h k l}^{n_{0}}\left[\mathrm{~J} / \mathrm{m}^{2}\right]$ & 0.72 & 0.74 & 1.11 & 0.80 & 0.85 \\
$E_{\text {ads }}^{n=1} / \mathrm{eV}$ & -1.92 & -2.18 & -2.18 & -2.25 & -2.38 \\
Site $(n=1)$ & $\mathrm{H}-\mathrm{B}$ & $3 \mathrm{f}-\mathrm{Lb}$ & $\mathrm{Tsb}-\mathrm{T} 2$ & $\mathrm{Qt}-\mathrm{Sb}$ & $\mathrm{Qt1}-\mathrm{Qt} 1$ \\
$d_{n=1}[\AA]$ & $1.72-2.35$ & $1.75-2.33$ & $1.63-2.33$ & $1.77-2.29$ & $1.80-2.34$ \\
\hline
\end{tabular}

those of the bare surfaces. It can as well be seen, that again, the size of the surface unit cell has an influence on $n_{0}$. Note that all the distances given in Table 4 agree very well with those of the single $\mathrm{H}$ and $\mathrm{Cl}$ ligands given in Tables 2 and 3.

For the case of the (111) surface and $n=n_{0}=3$, the $\mathrm{Cl}$ atoms adsorb at T2 with a slight horizontal offset towards T3. Two of the $\mathrm{H}$ atoms adsorb at Qt, one approximately at Qf. Due to the limited space available, however, they do not reside on top of the surface but have diffused into the region between the first and second ML of Fe. A similar rearrangement is observed for the (310) surface. Figure 8 shows the effect of the $\mathrm{HCl}$ adsorption onto the magnetization. One clearly sees a monotonous reduction with growing ligand adsorption for all surfaces. This is well explained as the sum of the changes of the individual adsorption species as shown in Figures 6 and 3. The increase in the (111) case, when going from two to three ligands, is similar to the adsorption of more than $4 \mathrm{H}$, as discussed above, and can be explained analogously. It is caused by the already mentioned changing position of the $\mathrm{H}$ atoms. In case of $n=3$ they 
Table 5. The minimum surface energy coverage values $n_{0}$ of $\mathrm{NH}_{3}$ (in molecules per $2 \times 2$ surface cell), the respective minimum surface energy $\gamma_{h k l}^{n_{0}}$, the adsorption energy of one molecule, the preferred adsorption sites, and the distance between the $\mathrm{N}$ and the topmost Fe atom in case of 1 ligand only (in case of $n=n_{0}$ the increase in $d$ is of maximal $0.03 \AA$.

\begin{tabular}{cccccc}
\hline$h k l$ & $(100)$ & $(110)$ & $(111)$ & $(211)$ & $(310)$ \\
\hline$n_{0}$ & 2 & 1 & 2 & 2 & 2 \\
$\gamma_{h k l}^{n_{0}}\left[\mathrm{~J} / \mathrm{m}^{2}\right]$ & 2.13 & 2.05 & 2.09 & 2.08 & 1.94 \\
$E_{\mathrm{ads}}^{n=1} / \mathrm{eV}$ & -0.53 & -0.51 & -0.85 & -0.74 & -0.74 \\
Site & $\mathrm{T}$ & $\mathrm{T}$ & $\mathrm{T} 1$ & $\mathrm{~T}$ & $\mathrm{~T} 1$ \\
$d_{n=1}[\AA]$ & 2.16 & 2.14 & 2.11 & 2.11 & 2.14 \\
\hline
\end{tabular}

are located between the first and second ML of Fe. Their interaction with the strongly magnetic first ML, thus, becomes weaker, whereas a possible growing reduction of the only slightly enhanced $m$ in the second ML will not have as strong an effect, see as well Figure 2.

Since the effect of the $\mathrm{HCl}$ adsorption at the Fe surfaces can be regarded as a superposition of the individual ligands, the absolute magnitude of the reduction of $m$ is similar to that of pure $\mathrm{H}$. This is because $\mathrm{Cl}$ adsorption does not alter the surface magnetization significantly, as discussed above. As Figure 8 shows, $m$ is reduced as well, but not below the bulk value.

\section{5. $\mathrm{NH}_{3}$ and $\mathrm{NH}_{4} \mathrm{Cl}$}

Although ammonia will adsorb only very weakly on iron surfaces during common synthesis conditions, the adsorption energy of $\mathrm{NH}_{3}$ on the Fe surfaces ranges from 0.51 to $-0.85 \mathrm{eV}$, indicating a preference for adsorption. [23] Thus, at low temperatures Fe NP may be covered by a significant amount of $\mathrm{NH}_{3}$. The resulting impact of this is depicted in Figure 9. It shows a slight decrease for the (100), (110), and (211) facets,

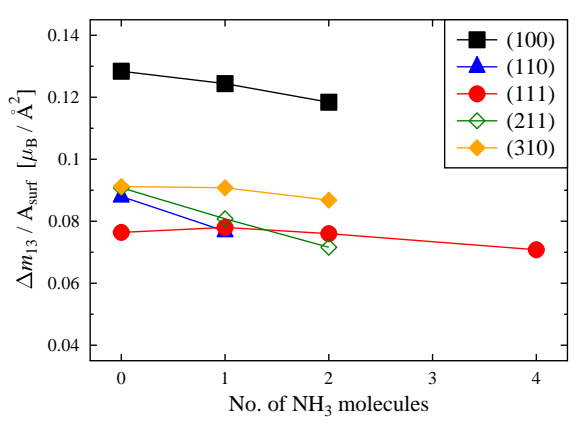

Figure 9. Magnetic moment of the three top Fe atoms upon $\mathrm{NH}_{3}$ adsorption, with respect to the bulk value, up to the coverage values given in Table 4.

whereas for the (111) and (310) surfaces the magnetization remains rather constant. 
The reason for these varying behaviours may be the different distributions of $m$ at the bare surfaces. For the (111) and (310) cases the part of the moment below the first layer is considerably larger than in the cases of the (100), (110), and (211) facets, see Figure 2. As these lower regions interact only little with the adsorbed $\mathrm{NH}_{3}$ due to the distance and the covalent character of the bond, the reducing effect of the adsorbates is weakened.

In any case, one can say that even if $\mathrm{NH}_{3}$ may adsorb at $\mathrm{Fe} \mathrm{NP}$ at low temperatures, the effect onto the NP surface magnetization will be very small.

Regarding the $\mathrm{NH}_{4} \mathrm{Cl}$ compound, we found that its dissociation into $\mathrm{NH}_{3}$ and $\mathrm{HCl}$ and the adsorption of $\mathrm{NH}_{3}, \mathrm{H}$, and $\mathrm{Cl}$ is energetically more favorable than the adsorption of the whole compound. [23] Considering the above mentioned weak adsorption of $\mathrm{NH}_{3}$ one can assume that the presence of ammonium molecules has the same effects on the surface magnetization as the presence of $\mathrm{HCl}$, which was already discussed above.

\section{6. $\mathrm{CH}_{3} \mathrm{COOH}$}

As in the case of $\mathrm{HCl}$ we found the configuration with separately adsorbed hydride and acetate to be the most stable one for all configurations. The $\mathrm{CH}_{3} \mathrm{COO}^{-}$adsorbs with its two $\mathrm{O}$ atoms on the surface, where the two $\mathrm{O}$ are located between two nearest neighbour Fe atoms in the upper most surface layer. The exact position of each $\mathrm{O}$ depends on the geometry of the specific surface. It is mainly influenced by the Coulomb repulsion between the $\mathrm{H}$ and the $\mathrm{O}$, which tends to maximize the distance between the two ligands. [23] The $\mathrm{H}$ adsorption site corresponds exactly to that of the $\mathrm{HCl}$ case as given in Table 4 for $n=1$.

Table 6 summarizes the adsorption energetics. One can see that the surface energies

Table 6. $\mathrm{CH}_{3} \mathrm{COOH}$ adsorption: the minimum surface energy coverage values $n_{0}$ (in molecules per $2 \times 2$ surface cell), the corresponding $\gamma_{h k l}^{n_{0}}$, the adsorption energy of one dissociated molecule, and the shortest distance $d(O-H)$ between an oxygen and the dissociated hydrogen.

\begin{tabular}{cccccc}
\hline$h k l$ & $(100)$ & $(110)$ & $(111)$ & $(211)$ & $(310)$ \\
\hline$n_{0}$ & 2 & 1 & 2 & 2 & 2 \\
$\gamma_{h k l}^{n_{0}}\left[\mathrm{~J} / \mathrm{m}^{2}\right]$ & 0.98 & 1.46 & 1.61 & 1.20 & 1.31 \\
$E_{\text {ads }}[\mathrm{eV}]$ & -1.73 & -1.36 & -1.97 & -1.87 & -2.14 \\
$d(O-H)[\AA]$ & 2.98 & 2.61 & 3.29 & 3.30 & 3.55 \\
\hline
\end{tabular}

strongly depend on the distance between the adsorbed acetate and hydride. $E_{\text {ads }}$ is most pronounced for the (310) surface where $d(O-H)$ is largest. Contrary, it is weakest for the smallest distance in the (110) case. Due to the large volume of the acetate the coverage values leading to the lowest surface energies, $n_{0}$, are two, with the exception of the (110) case, where $n_{0}=1$ holds.

The effect on the surface magnetism is shown in Figure 10. The qualitative change is comparable to the $\mathrm{HCl}$ case, however, the magnitude is considerably smaller. As 


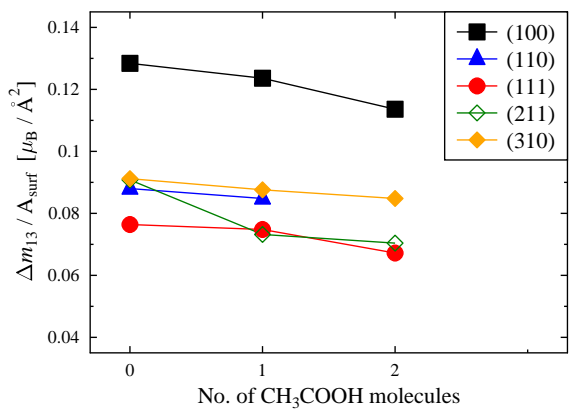

Figure 10. Magnetic moment of the three top $\mathrm{Fe}$ atoms upon $\mathrm{HCl}$ adsorption, with respect to the bulk value, up to the coverage values given in Table 4.

the behavior of the magnetization in the case of $\mathrm{HCl}$ could be well explained by a superposition of the isolated $\mathrm{H}$ and $\mathrm{Cl}$, with $\mathrm{H}$ being responsible for the net reduction, this seems to be as well the case for the $\mathrm{CH}_{3} \mathrm{COOH}$ adsorption. At the same time the quantitatively smaller effect hints to an weakened coupling between the $\mathrm{H}$ and the $\mathrm{Fe}$ atoms, which is probably caused by the larger concentration of ligand atoms at the surface.

\section{Summary and Conclusions}

We have investigated the surface magnetism of five different iron surfaces of low surface energy and its behavior upon the adsorption of $\mathrm{H}, \mathrm{Cl}, \mathrm{HCl}, \mathrm{CH}_{3} \mathrm{COOH}, \mathrm{NH}_{3}$, and $\mathrm{NH}_{4} \mathrm{Cl}$. For the bare surfaces we found that the (100) facet has the highest $m$ per surface area. Upon $\mathrm{H}$ adsorption, the magnetization of all surfaces is lowered proportionally to the number of adsorbed ligands. The highest resulting $m$ per area in the case of the minimum surface energy is then found at the (310) facet, which is nevertheless only slightly above that of the (100) facet. In the case of $\mathrm{Cl}$ adsorption we have found that the surface magnetization of all surfaces is altered only very slightly, leaving the (100) surface with the highest $m / A$ ratio. If $\mathrm{HCl}$ is adsorbed, it is dissociated and the $\mathrm{H}$ and the $\mathrm{Cl}$ adsorb separately as single ligands. Their effect on $m$ can be described as a superposition of that of the single isolated $\mathrm{H}$ and $\mathrm{Cl}$ adsorbates. As the number of possible $\mathrm{H}$ ligands is smaller than in the case of pure $\mathrm{H}$ adsorption, which is due to the presence of $\mathrm{Cl}$, the (100) surface remains the one with the highest $m$ per area at the minimum surface energy configuration. The adsorption of $\mathrm{NH}_{3}$ does not lead to significant energy gain and will, therefore, not affect the magnetization in experiments. $\mathrm{NH}_{4} \mathrm{Cl}$ dissociates into $\mathrm{NH}_{3}$, and $\mathrm{H}$, and $\mathrm{Cl}$ and will, thus, affect the surface magnetization in an alike manner as $\mathrm{HCl}$.

Thus, we conclude that cubic Fe NP, as found and synthesized in previous experiments, should show improved magnetic properties compared to those of other morphology, as their $m$ is generally most strongly increased. Experimental findings 
of Fe NP with average magnetizations below the Fe bulk value, however, can not be explained by assuming adsorption of the within this work investigated species on infinitely extended surfaces, as all surfaces with ligands still have improved $m$ at the surface. Assuming $\mathrm{H}$ atoms underneath the surface does not alter this result. The reason for the discrepancy between theoretical and experimental findings may, thus, be other ligands not considered in the present work, or possible effects that are neglected in our approach, for example by assuming $T=0 \mathrm{~K}$, not considering non-collinear magnetism and, thus, possible spin reorientation processes due to surface stress changes, [42], or assuming infinitely extended surfaces.

\section{Acknowledgments}

The authors thank the ANR for the financial support through the funded project INANOPROCE (Projet ANR-11-JS10-0007). They also thank the CALcul en MIdiPyrénés (CALMIP, grant 2013/2014-P0843) for generous allocation of computer time. Part of this work was also performed using HPC resources from GENCI-CINES (Grant 2013/2014-096940), GENCI-CCRT (Grant 2013/2014-096940) and GENCIIDRIS (Grant 2013/2014-096940).

\section{References}

[1] S. D. Bader. Colloquium: Opportunities in nanomagnetism. Rev. Mod. Phys., 78:1-15, Jan 2006.

[2] Anna Moisala, Albert G Nasibulin, and Esko I Kauppinen. The role of metal nanoparticles in the catalytic production of single-walled carbon nanotubes a review. Journal of Physics: Condensed Matter, 15(42):S3011, 2003.

[3] You Qiang, J. Antony, M.G. Marino, and S. Pendyala. Synthesis of core-shell nanoclusters with high magnetic moment for biomedical applications. Magnetics, IEEE Transactions on, 40(6):3538-3540, 2004.

[4] Q. A. Pankhurst, J. Connolly, S. K. Jones, and J. Dobson. Applications of magnetic nanoparticles in biomedicine. Journal of Physics D: Applied Physics, 36(13):R167, 2003.

[5] D. L. Huber. Synthesis, properties, and applications of iron nanoparticles. Small, 1(5):482-501, 2005.

[6] C L Dennis, A J Jackson, J A Borchers, P J Hoopes, R Strawbridge, A R Foreman, J van Lierop, C Grttner, and R Ivkov. Nearly complete regression of tumors via collective behavior of magnetic nanoparticles in hyperthermia. Nanotechnology, 20(39):395103, 2009.

[7] L.-M. Lacroix, S. Lachaize, A. Falqui, T. Blon, J. Carrey, M. Respaud, F. Dumestre, C. Amiens, O. Margeat, B. Chaudret, P. Lecante, and E. Snoeck. Ultrasmall iron nanoparticles: Effect of size reduction on anisotropy and magnetization. Journal of Applied Physics, 103(7):07D521, 2008.

[8] Markus Ernst Gruner and Peter Entel. Simulating functional magnetic materials on supercomputers. Journal of Physics: Condensed Matter, 21(29):293201, 2009.

[9] Isabelle M. L. Billas, J. A. Becker, A. Châtelain, and Walt A. de Heer. Magnetic moments of iron clusters with 25 to 700 atoms and their dependence on temperature. Phys. Rev. Lett., 71:4067-4070, Dec 1993.

[10] M. Niemeyer, K. Hirsch, V. Zamudio-Bayer, A. Langenberg, M. Vogel, M. Kossick, C. Ebrecht, K. Egashira, A. Terasaki, T. Möller, B. v. Issendorff, and J. T. Lau. Spin coupling and orbital angular momentum quenching in free iron clusters. Phys. Rev. Lett., 108:057201, Jan 2012. 
[11] O. Šipr, M. Košuth, and H. Ebert. Magnetic structure of free iron clusters compared to iron crystal surfaces. Phys. Rev. B, 70:174423, Nov 2004.

[12] Murilo L. Tiago, Yunkai Zhou, M. M. G. Alemany, Yousef Saad, and James R. Chelikowsky. Evolution of magnetism in iron from the atom to the bulk. Phys. Rev. Lett., 97:147201, Oct 2006.

[13] J. A. Franco, A. Vega, and F. Aguilera-Granja. Average magnetization and local magnetic moments of fe ${ }_{N}$ clusters $(n<230)$. Phys. Rev. B, 60:434-439, Jul 1999.

[14] S. Polesya, O. Šipr, S. Bornemann, J. Minár, and H. Ebert. Magnetic properties of free fe clusters at finite temperatures from first principles. EPL (Europhysics Letters), 74(6):1074, 2006.

[15] P. Błoński and A. Kiejna. Structural, electronic, and magnetic properties of bcc iron surfaces. Surface Science, 601(1):123 - 133, 2007.

[16] Dale L Huber, Eugene L Venturini, James E Martin, Paula P Provencio, and Rina J Patel. Synthesis of highly magnetic iron nanoparticles suitable for field structuring using a -diketone surfactant. Journal of Magnetism and Magnetic Materials, 278(3):311 - 316, 2004.

[17] Min Chen, Saeki Yamamuro, Dorothy Farrell, and Sara A. Majetich. Gold-coated iron nanoparticles for biomedical applications. Journal of Applied Physics, 93(10):7551-7553, 2003.

[18] Frédéric Dumestre, Bruno Chaudret, Catherine Amiens, Philippe Renaud, and Peter Fejes. Superlattices of Iron Nanocubes Synthesized from Fe[N(SiMe3)2]2. Science, 303(5659):821-823, 2004.

[19] Anca Meffre, Sebastien Lachaize, Christophe Gatel, Marc Respaud, and Bruno Chaudret. Use of long chain amine as a reducing agent for the synthesis of high quality monodisperse iron(0) nanoparticles. J. Mater. Chem., 21:13464-13469, 2011.

[20] Anastasia V. Trunova, R. Meckenstock, I. Barsukov, C. Hassel, O. Margeat, M. Spasova, J. Lindner, and M. Farle. Magnetic characterization of iron nanocubes. Journal of Applied Physics, 104(9):-, 2008.

[21] Vinciane Kelsen, Bianca Wendt, Svenja Werkmeister, Kathrin Junge, Matthias Beller, and Bruno Chaudret. The use of ultrasmall iron(0) nanoparticles as catalysts for the selective hydrogenation of unsaturated C-C bonds. Chem. Commun., 49:3416-3418, 2013.

[22] J. Carvell, E. Ayieta, A. Gavrin, Ruihua Cheng, V. R. Shah, and P. Sokol. Magnetic properties of iron nanoparticle. Journal of Applied Physics, 107(10):103913, 2010.

[23] Guntram Fischer, Romuald Poteau, Sébastien Lachaize, and Iann C. Gerber. Surfaces of colloidal iron nanoparticle in its chemical environment: a dft description. Langmuir, (http://dx.doi.org/10.1021/la502963n):just accepted, 2014.

[24] P. Hohenberg and W. Kohn. Inhomogeneous electron gas. Phys. Rev., 136(3B):B864-B871, Nov 1964.

[25] W. Kohn and L. J. Sham. Self-consistent equations including exchange and correlation effects. Phys. Rev., 140(4A):A1133-A1138, Nov 1965.

[26] John P. Perdew, Kieron Burke, and Matthias Ernzerhof. Generalized gradient approximation made simple. Phys. Rev. Lett., 77:3865-3868, Oct 1996.

[27] G. Kresse and J. Hafner. Ab initio molecular dynamics for liquid metals. Phys. Rev. B, 47:558, 1993.

[28] G. Kresse and J. Furthmüller. Efficiency of ab-initio total energy calculations for metals and semiconductors using a plane-wave basis set. Comput. Mat. Sci., 6:15, 1996.

[29] P. E. Blöchl. Projector augmented-wave method. Phys. Rev. B, 50(24):17953-17979, Dec 1994.

[30] Charles Kittel. Physique de l' état solide. Bordas, Paris, 5 edition, 1983.

[31] D. E. Jiang and Emily A. Carter. Diffusion of interstitial hydrogen into and through bcc Fe from first principles. Phys. Rev. B, 70:064102, Aug 2004.

[32] R. F. W. Bader. Atoms in Molecules: A Quantum Theory. Oxford University Press, 1990.

[33] W. Tang, E. Sanville, and G. Henkelman. A grid-based bader analysis algorithm without lattice bias. Journal of Physics: Condensed Matter, 21(8):084204, 2009.

[34] D. M. Wood and A. Zunger. A new method for diagonalising large matrices. Journal of Physics 
A: Mathematical and General, 18(9):1343, 1985.

[35] M. Digne, P. Sautet, P. Raybaud, P. Euzen, and H. Toulhoat. Hydroxyl groups on -alumina surfaces: A dft study. Journal of Catalysis, 211(1):1 - 5, 2002.

[36] Michelle J.S. Spencer, Andrew Hung, Ian K. Snook, and Irene Yarovsky. Density functional theory study of the relaxation and energy of iron surfaces. Surface Science, 513(2):389 - 398, 2002.

[37] P. Błoński and A. Kiejna. Calculation of surface properties of bcc iron. Vacuum, 74(2):179 - 183, 2004. Proceedings of the International Workshop on Surface Physics: Metals on Solid Surfaces.

[38] V. B. Chikarmane and K. Schrder. The effect of hydrogen on the magnetization of iron films. $J$. Phys. Colloques, 49:1737, Dec 1988.

[39] G. J. Mankey, M. T. Kief, F. Huang, and R. F. Willis. Hydrogen chemisorption on ferromagnetic thin film surfaces. volume 11, pages 2034-2039. AVS, 1993.

[40] Mark B. Knickelbein. Adsorbate-induced enhancement of the magnetic moments of iron clusters. Chemical Physics Letters, 353(34):221 - 225, 2002.

[41] R Fournier and D.R Salahub. Chemisorption and magnetization: a bond order-rigid band model. Surface Science, 238(13):330 - 340, 1990.

[42] Dirk Sander, Zhen Tian, and Jürgen Kirschner. The role of surface stress in structural transitions, epitaxial growth and magnetism on the nanoscale. Journal of Physics: Condensed Matter, 21(13):134015, 2009. 